\title{
A Paintability Version of the Combinatorial Nullstellensatz, and List Colorings of $k$-partite $k$-uniform Hypergraphs
}

\author{
Uwe Schauz \\ Department of Mathematics and Statistics \\ King Fahd University of Petroleum and Minerals \\ Dhahran 31261, Saudi Arabia \\ schauz@kfupm.edu.sa
}

Submitted: May 15, 2010; Accepted: Dec 2, 1010; Published: Dec 10, 2010

Mathematics Subject Classifications: 05C15, 11C08, 91A43, 05C65, 05C50

\begin{abstract}
We study the list coloring number of $k$-uniform $k$-partite hypergraphs. Answering a question of Ramamurthi and West, we present a new upper bound which generalizes Alon and Tarsi's bound for bipartite graphs, the case $k=2$. Our results hold even for paintability (on-line list colorability). To prove this additional strengthening, we provide a new subject-specific version of the Combinatorial Nullstellensatz.
\end{abstract}

\section{Introduction}

We examine list colorability (choosability) of hypergraphs $\mathcal{H}=(V, \mathcal{E})$. For a fixed tuple $L=\left(L_{v}\right)_{v \in V}$ of color lists (sets), the hypergraph $\mathcal{H}$ is called $L$-colorable if there exist a vertex coloring $v \longmapsto c(v) \in L_{v}$ without monochromatic edges $e \in \mathcal{E}$, i.e. $|c(e)|>1$. For a fixed tuple $\ell=\left(\ell_{v}\right)_{v \in V} \in \mathbb{Z}_{>0}^{V}$, the hypergraph $\mathcal{H}$ is called $\ell$-list colorable $(\ell$-choosable $)$ if it is $L$-colorable for any tuple $L$ of color lists $L_{v}$ with cardinalities $\left|L_{v}\right|=\ell_{v}$. As generalization of ordinary graph colorings, with just one common list of available colors for all vertices, list colorings were introduced by Vizing [Viz] and independently by Erdös, Rubin and Taylor [ERT]. They worked with usual graphs, i.e. 2-uniform hypergraphs $(|e|=2$ for all $e \in \mathcal{E})$, and proved that Brooks' Theorem about the maximal degree as upper bound for the required number of colors holds in the more general setting of list colorings. Other theorems about usual colorings could be generalized later as well, see [Tu, Al, KTV]. One could think that if we can color a graph with $k$ colors then it should be $k$-list colorable, i.e $(k, k, \ldots, k)$-list colorable; "making habitats less overlapping should 
help to avoid collisions". However, this is not the case, surprisingly, it can be more difficult to color a graph if the lists $L_{v}$ are different. Erdös, Rubin and Taylor [ERT] showed in [ERT] that even graphs that are colorable with just 2 colors (i.e. bipartite graphs) can have arbitrarily big list chromatic numbers. Therefore, one had to ask how big the lists have to be in order to guarantee the existence of colorings. The general upper bound provided by Brooks' Theorem turns out to be far from tight in many cases. In fact, Alon and Tarsi could provide in [AlTa, Theorem 3.2] a much better one. In particular, their result implies that bipartite graphs with maximal degree $2 k$ are $(k+1)$-list colorable. In Section 2, we generalize Alon and Tarsi's results about list colorability of bipartite graphs (2-partite 2-uniform hypergraphs) to $k$-partite $k$-uniform hypergraphs. Ramamurthi and West asked at the end of their paper [RaWe] for such a hypergraph analog of Alon and Tarsi's result.

In the course of the described development Alon and Tarsi found the so called Polynomial Method and the Combinatorial Nullstellensatz [Al2, Scha1], which is of fundamental importance inside and beyond combinatorics:

Theorem 1.1 (Combinatorial Nullstellensatz). If $X_{1}^{\alpha_{1}} X_{2}^{\alpha_{2}} \cdots X_{n}^{\alpha_{n}}$ occurs as monomial of maximal degree in a polynomial $P\left(X_{1}, X_{2}, \ldots, X_{n}\right)$, then this polynomial has a nonzero in any domain $L_{1} \times L_{2} \times \cdots \times L_{n}$ with $\left|L_{j}\right|>\alpha_{j}$ for $j=1,2, \ldots, n$.

A simple application of this theorem led to the so called Alon-Tarsi List Coloring Theorem [AlTa, Theorem 1.1], which was Alon and Tarsi's main tool in the verification of their upper bound for the list chromatic number of bipartite graphs. This theorem on its own achieved some prominence in the theory of list colorings, and the upper bound of the list chromatic number for bipartite graphs is just one of its many repercussions. Accordingly, it seems natural to generalize the Alon-Tarsi Theorem to hypergraphs in order to generalize its repercussions, and, in particular, the upper bound for bipartite graphs. This is exactly what Ramamurthi and West tried in [RaWe]. They actually found a hypergraph extension of the Alon-Tarsi Theorem, but this extension did not help on with the upper bound. The generalized Alon-Tarsi Condition about certain generalized Eulerian subgraphs in their result is too hard to verify. Therefore, we replaced this condition with the condition that a certain $\alpha$-permanent $\operatorname{per}_{\alpha}(A)$ of a so called zero rowsum incidence matrices $A$ has to be different from zero (Theorem 2.1). In the setting of $k$-uniform $k$-partite hypergraphs, such a zero row-sum incidence matrices matrix $A$ can be obtained from the usual 0-1 incidence matrix $A^{\prime}$ by multiplying its columns with appropriate scalars. Therefore, we are left with the study of $\operatorname{per}_{\alpha}\left(A^{\prime}\right)$, which turns out to be the number of $\alpha$-orientations. Summarizing, we see that, for $k$-uniform $k$-partite hypergraphs, the mere existence of $\alpha$-orientations assures proper list colorings (Theorem 2.2). With this main result of Section 1, our verification of proper list colorings in Section 2 is reduced to hunting down good orientations.

Moreover, we discovered in [Scha2] an additional further generalization of the concept of list colorings. This generalization is based on a different point of view. Instead of assigning color lists $L_{v}$ of size $\ell_{v}$ to the vertices $v$ of a (hyper)graph, we assign sets of vertices $V_{1}, V_{2}, \ldots \subseteq V$ to colors (say $c_{1}, c_{2}, \ldots$ ) such that each vertex $v$ is contained in 
exactly $\ell_{v}$ sets $V_{i}$. The $i^{\text {th }}$ set $V_{i}$ describes the range of vertices that are allowed to receive the $i^{\text {th }}$ color $c_{i}$. Both concepts of restricting the availability of colors are equivalent, but the second one can be generalized as follows. When we have already used the first $i$ colors $c_{1}, c_{2}, \ldots, c_{i}$ in a coloration process, then we allow to change the vertex sets $V_{j}$ with $j>i$, only the property that each vertex $v$ is contained in exactly $\ell_{v}$ lists shall remain. Such changes on the flight may be required in real-life applications. Actually, we showed in [Scha3] that this concept has applications in time scheduling. We also saw that $\ell$-list colorable graphs not always are $\ell$-paintable, as we say, i.e., there does not have to be a winning coloration strategy if the vertex sets $V_{i}$ are allowed to change (see also [Zhu, Theorem 14\&15]). Therefore, it is quite surprising that almost all theorems about list colorings still hold in the new framework of paintability (on-line list colorability), see [Scha2, Scha3, HKS]. What is with the results of this paper? Well, all our results extend, just replace every occurrence of "list color..." with "paint..." and everything is fine. In order to achieve this additional strengthening we need a strengthened version of the Combinatorial Nullstellensatz (Theorem 1.1), which we provide with Theorem 4.5. As we will see at the end of the paper, this is not possible without additional assumptions. The additional assumption that we found is to only allow substitutions of algebraically independent elements into the polynomial. This restriction is quite strong and will make the theorem useless for most applications. However, when it comes to coloration problems we may even use symbolic variables as colors, so that we get algebraic independence for free. All this is worked out in a more game-theoretic setting in Section 4. The definitions and proofing ideas in this section generalize the introduction of paintability of graphs in [Scha2] and the purely combinatorial proof of the Alon-Tarsi List Coloring Theorem in [Scha3]. We also point out that, beside our paintability strengthening of the Combinatorial Nullstellensatz, other versions of this theorem may lead to other improvements of list coloration theorems. The "Quantitative" Combinatorial Nullstellensatz [Scha1, Theorem 3.3 $(i)$ ] is one such example, although the relatively technical "quantitative" results only become handsome in special situations like those in [Scha1, Section 5].

\section{Alon and Tarsi's Theorem for Hypergraphs}

In this section we provide our tool for detecting colorings of hypergraphs $\mathcal{H}=(V, \mathcal{E})$. We $\mathcal{H}=(V, \mathcal{E})$ always work over integral domains $\mathcal{R}$. A matrix $A=\left(a_{e v}\right) \in \mathcal{R}^{\mathcal{E} \times V}$ with

$$
a_{e v} \neq 0 \Longleftrightarrow v \in e
$$

and with vanishing row-sums,

$$
\sum_{v \in e} a_{e v}=0 \quad \text { for all } e \in \mathcal{E}
$$

is a zero row-sum incidence matrix of $\mathcal{H}$. The homogenous polynomial

$$
P_{A}:=\prod_{e \in \mathcal{E}} \sum_{v \in V} a_{e v} X_{v}
$$


is the matrix polynomial of $A$ over $R$. We examine its nonzeros and coefficents:

- The nonzeros $x=\left(x_{v}\right)_{v \in V}$ of $P_{A}$ give rice to proper vertex colorings $v \mapsto x_{v}$ of $\mathcal{H}$. Conversely, if the vertex colors $x_{v}$ of a coloring $v \mapsto x_{v}$ of $\mathcal{H}$ lie in an extension ring $\widehat{\mathcal{R}}$ of $R$, and are algebraically independent over $\mathcal{R}$, then $x=\left(x_{v}\right)_{v \in V}$ is a nonzero of $P_{A}$. Furthermore, if $\widehat{\mathcal{R}}$ is an extension ring of $R$ and $L_{v} \subseteq \widehat{\mathcal{R}}$ for all $v \in V$, then the colors $x_{v}$ of the vertices $v \in V$ lies in the lists $L_{v}$ if and only if the nonzero $x$ of $P_{A}$ lies in the grid $\prod_{v \in V} L_{v} \subseteq \widehat{\mathcal{R}}^{V}$. Therefore, any list coloring problem can be modelled by a polynomial function on a grid. Finding a suitable ring extension $\widehat{\mathcal{R}}$ as working environment is no problem. Without loss of generality, we may view all the colors in all the given color lists $L_{v}$ as symbolic variables, and take $\widehat{\mathcal{R}}$ as the polynomial ring in these variables over $\mathcal{R}$.

- The coefficient $\left(P_{A}\right)_{\alpha}$ of $X^{\alpha}=\prod_{v} X_{v}^{\alpha_{v}}$ in $P_{A}$ is given by the $\alpha$-permanent of $A$,

$$
\left(P_{A}\right)_{\alpha}=\operatorname{per}_{\alpha}(A):=\sum_{\substack{\sigma: \mathcal{E} \rightarrow V \\\left|\sigma^{-1}(v)\right|=\alpha_{v}}} \prod_{e \in \mathcal{E}} a_{e, \sigma(e)}
$$

This kind of permanent has the property that

$$
\operatorname{per}_{\alpha}(A)=0 \quad \text { if } \sum_{v \in V} \alpha_{v} \neq|\mathcal{E}|
$$

which is also reflected in the homogeneity of $P_{A}$. It is related to the usual permanent per $:=\operatorname{per}_{1}=\operatorname{per}_{(1,1, \ldots, 1)}$ by the equation

$$
\left(\prod_{v \in V} \alpha_{v} !\right) \operatorname{per}_{\alpha}(A)=\operatorname{per}(A\langle\mid \alpha\rangle) \quad \text { if } \sum_{v \in V} \alpha_{v}=|\mathcal{E}|
$$

where $A\langle\mid \alpha\rangle$ is a matrix that contains the column of $A$ with index $v$ exactly $\alpha_{v}$ times (as in [AlTa2] or [Scha1, Definition 5.2]).

Summarizing and paraphrasing, nonzeros are colorings, and coefficients are permanents. The gap between coefficients and nonzeros is being closed by the Combinatorial Nullstellensatz (Theorem 1.1), as in homogenous polynomials all monomials have maximal degree. We obtain:

Theorem 2.1. Let $A$ be a zero row-sum incidence matrix of a hypergraph $\mathcal{H}=(V, \mathcal{E})$. Then, for $\alpha \in \mathbb{N}^{V}$, holds

$$
\operatorname{per}_{\alpha}(A) \neq 0 \Longrightarrow \mathcal{H} \text { is }(\alpha+1) \text {-list colorable. }
$$

Apparently, the $\alpha$-permanent in this theorem is a sum running over all, so called, $\alpha$-orientations $\varphi: \mathcal{E} \longrightarrow V, e \longmapsto e^{\varphi} \in e$ of $\mathcal{H}$, i.e., those orientations with score sequence $d^{\varphi}:=\left(d^{\varphi}(v)\right)_{v \in V}$ equal to $\alpha$,

$$
d^{\varphi}=\alpha \quad \text { where } \quad d^{\varphi}(v):=\left|\varphi^{-1}(v)\right| .
$$

Using this terminology, we can prove the following theorem, which for $k=2$ was first proven in Alon and Tarsi's paper [AlTa]: 
Theorem 2.2. Let $\mathcal{H}$ be a $k$-partite $k$-uniform hypergraph. If there exists an $\alpha$-orientation of $\mathcal{H}$, then $\mathcal{H}$ is $(\alpha+1)$-list colorable.

Proof. Let $A^{\prime}=\left(a_{e v}^{\prime}\right) \in\{0,1\}^{\mathcal{E} \times V}$ be the 0 -1 incidence matrix of $\mathcal{H}$ over the integers, and let $\varphi: \mathcal{E} \longrightarrow V$ be an $\alpha$-orientation of $\mathcal{H}$. Then

$$
\operatorname{per}_{\alpha}\left(A^{\prime}\right) \neq 0
$$

as

$$
\prod_{e \in \mathcal{E}} a_{e, \varphi(e)}^{\prime}=1>0
$$

and the other summands in the definition of per $_{\alpha}$ are nonnegative. Now, let $\varepsilon_{1}, \varepsilon_{2}, \ldots, \varepsilon_{k}$ be nonzero numbers with

$$
\varepsilon_{1}+\varepsilon_{2}+\cdots+\varepsilon_{k}=0
$$

one $\varepsilon_{i}$ for each partition class $V_{i}$ of $\mathcal{H}$. Multiplying the columns $a_{*, v}^{\prime}$ that correspond to vertices $v$ of the $i^{\text {th }}$ partition class $V_{i}$ of $\mathcal{H}$ with $\varepsilon_{i}$ (for $i=1, \ldots, k$ ), we obtain a matrix $A$ with the properties required in Theorem 2.1, so that $\mathcal{H}$ is $(\alpha+1)$-list colorable. This is so since each edge $e$ of $\mathcal{H}$ has exactly one vertex in each partition class $V_{i}$ of $\mathcal{H}$, so that $A$ has zero row-sums; and $\operatorname{per}_{\alpha}(A) \neq 0$ since if a column $a_{*, v}^{\prime}$ of a matrix $A^{\prime}$ is multiplied by $\varepsilon_{i}$, its $\alpha$-permanent will multiply by $\varepsilon_{i}^{\alpha_{v}}$.

\section{List Colorability of $k$-uniform $k$-partite Hypergraphs}

In this section, we only consider nontrivial hypergraphs $\mathcal{H}$, i.e., we always assume $\mathcal{E}(\mathcal{H}) \neq$ $\varnothing$. We search for certain good orientations of hypergraphs. This will lead to good upper bounds for the list chromatic number of $k$-uniform $k$-partite hypergraphs $\mathcal{H}$, i.e., the smallest $m \in \mathbb{N}$ for which $\mathcal{H}$ is $m$-list colorable, i.e. $(m, m, \ldots, m)$-list colorable.

Our observations and results are based on the following definition, involving partial hypergraphs $H \leq \mathcal{H}$, i.e. $\mathcal{E}(H) \subseteq \mathcal{E}(\mathcal{H})$ and $\varnothing \neq V(H) \subseteq V(\mathcal{H})$ :

\section{Definition 3.1.}

$H \leq \mathcal{H}$

$$
L(\mathcal{H}):=\max _{H \leq \mathcal{H}} \frac{|\mathcal{E}(H)|}{|V(H)|} \quad \text { and } \quad \check{L}(\mathcal{H}):=\max _{H \leq \mathcal{H}} \frac{|\mathcal{E}(H)|-1}{|V(H)|}
$$

Why are these two parameters of interest in our search for good orientations? Well, actually we want to ascertain the existence of orientations $\varphi: \mathcal{E} \longrightarrow V$ of $\mathcal{H}$ with small $\varphi$-scores $d_{\mathcal{H}}^{\varphi}(v)$, which is defined, a bit more general, for arbitrary partial hypergraphs $H \leq \mathcal{H}$, by

$$
d_{H}^{\varphi}(v):=\left|\varphi^{-1}(v) \cap \mathcal{E}(H)\right|=\left|\left(\left.\varphi\right|_{\mathcal{E}(H)}\right)^{-1}(v)\right| .
$$

In particular, the maximal $\varphi$-score

$$
\Delta(\varphi):=\max _{v \in V} d_{\mathcal{H}}^{\varphi}(v)
$$

should be as small as possible. Now, both $L(\mathcal{H})$ and $\check{L}(\mathcal{H})+1$ describe this smallest possible value. The optimum is given by rounding up to $\lceil L(\mathcal{H})\rceil$, respectively down to 
$\lfloor\check{L}(\mathcal{H})+1\rfloor$. In fact, we easily see that $L(\mathcal{H})$ is a lower bound. For all orientations $\varphi: \mathcal{E} \longrightarrow V$ we have

$$
\Delta(\varphi)=\max _{H \leq \mathcal{H}} \max _{v \in V(H)} d_{H}^{\varphi}(v) \geq \max _{H \leq \mathcal{H}} \underset{v \in V(H)}{\operatorname{average}} d_{H}^{\varphi}(v)=L(\mathcal{H})
$$

since

$$
|\mathcal{E}(H)|=\sum_{v \in V(H)} d_{H}^{\varphi}(v)
$$

More surprising is that the value $\lceil L(\mathcal{H})\rceil$ actually can be achieved, and that this number equals the value $\lfloor\check{L}(\mathcal{H})+1\rfloor$ :

Lemma 3.2. Each hypergraph $\mathcal{H}=(V, \mathcal{E})$ has an orientation $\varphi: \mathcal{E} \longrightarrow V$ with

$$
\Delta(\varphi)=\lceil L(\mathcal{H})\rceil=\lfloor\check{L}(\mathcal{H})+1\rfloor .
$$

Proof. We basically follow the proof of the graph-theoretic analog in [AlTa, Lemma 3.1]. Let

$$
m:=\lfloor\check{L}(\mathcal{H})+1\rfloor>\check{L}(\mathcal{H}) .
$$

We construct a bipartite graph $B^{m}$ as follows. For each hyperedge $e \in \mathcal{E}$, we introduce a vertex $\bar{e}$, and corresponding to each vertex $v \in V$ of $\mathcal{H}$ we introduce another $m$ vertices $(v, 1),(v, 2), \ldots,(v, m)$. Then, we connect a vertex $\bar{e}$ in the first part $\overline{\mathcal{E}} \subseteq V\left(B^{m}\right)$ with a vertex $(v, i)$ in the second part $V\left(B^{m}\right) \backslash \overline{\mathcal{E}}$ if and only if $e \ni v$ in $\mathcal{H}$. Now, it is sufficient to find a matching of $\overline{\mathcal{E}}$ in $B^{m}$. Such a matching $\bar{e} \longmapsto\left(v_{\bar{e}}, i_{\bar{e}}\right)$ would induce an orientation $\varphi: \mathcal{E} \longrightarrow V$ of $\mathcal{H}$ via

$$
e \longmapsto \bar{e} \longmapsto\left(v_{\bar{e}}, i_{\bar{e}}\right) \longmapsto v_{\bar{e}}=: \varphi(e),
$$

with maximal score at most $m$, so that

$$
\lceil L(\mathcal{H})\rceil \stackrel{(13)}{\leq} \Delta(\varphi) \leq m=\lfloor\check{L}(\mathcal{H})+1\rfloor \leq\lceil L(\mathcal{H})\rceil
$$

and the lemma would follow. However, the existence of such a matching follows from Hall's Theorem. We only have to show that each nonempty subset $\bar{E} \subseteq \overline{\mathcal{E}}$ has more than $|\bar{E}|-1$ neighbors in $B^{m}$. To this end, let $E \subseteq \mathcal{E}$ be the set of edges in $\mathcal{H}$ corresponding to $\bar{E} \subseteq \overline{\mathcal{E}}$. Let $\mathcal{H}[E] \leq \mathcal{H}$ be its induced partial hypergraph, and let $\bigcup E=V(\mathcal{H}[E]) \subseteq V$ be the set of all end-vertices of edges in $E$. Then, indeed, the number of neighbors of $\bar{E}$ in $B^{m}$ is

$$
m|\bigcup E|>\check{L}(\mathcal{H})|\bigcup E| \geq \frac{|\mathcal{E}(\mathcal{H}[E])|-1}{|V(\mathcal{H}[E])|} \| U E|=| \bar{E} \mid-1
$$

Note that it can be advantageous to use the second expression $\lfloor\check{L}(\mathcal{H})+1\rfloor$, instead of $\lceil L(\mathcal{H})\rceil$, when one wants to utilize an upper bound for $L(\mathcal{H})$. We will see this at the end of the section. Actually, it can be difficult to calculate $L(\mathcal{H})$ or $\check{L}(\mathcal{H})$ so that upper bounds have to be used. We will employ the following one: 
Lemma 3.3. Let $\mathcal{H}=\left(V_{1} \uplus V_{2} \uplus \cdots \uplus V_{k}, \mathcal{E}\right)$ be a $k$-partite $k$-uniform hypergraph with parts $V_{1}, V_{2}, \ldots, V_{k}$. Let $\Delta(\mathcal{H}):=\max _{v \in V} d(v)$ be the maximal degree of $\mathcal{H}$, and let $\Delta_{i}(\mathcal{H}):=$ $\max _{v \in V_{i}} d(v)$ be the maximal degree inside $V_{i}(i=1, \ldots, k)$. Then

$$
L(\mathcal{H}) \leq \frac{1}{1 / \Delta_{1}(\mathcal{H})+1 / \Delta_{2}(\mathcal{H})+\cdots+1 / \Delta_{k}(\mathcal{H})} \leq \frac{1}{k} \Delta(\mathcal{H})
$$

Proof. Since $\mathcal{E} \neq \varnothing$, we may allow in the definition of $L(\mathcal{H})$, and in the minima in the following part of this proof, only subgraphs $H$ with $\mathcal{E}(H) \neq \varnothing$, and can conclude as follows:

$$
\begin{aligned}
\frac{1}{L(\mathcal{H})} & =\min _{H \leq \mathcal{H}} \frac{|V(H)|}{|\mathcal{E}(H)|} \\
& \geq \min _{H \leq \mathcal{H}} \frac{\left|V(H) \cap V_{1}\right|}{|\mathcal{E}(H)|}+\min _{H \leq \mathcal{H}} \frac{\left|V(H) \cap V_{2}\right|}{|\mathcal{E}(H)|}+\cdots+\min _{H \leq \mathcal{H}} \frac{\left|V(H) \cap V_{k}\right|}{|\mathcal{E}(H)|} \\
& =\frac{1}{\Delta_{1}(\mathcal{H})}+\frac{1}{\Delta_{2}(\mathcal{H})}+\cdots+\frac{1}{\Delta_{k}(\mathcal{H})} \\
& \geq \frac{k}{\Delta(\mathcal{H})} .
\end{aligned}
$$

We want to go a little bit more into detail and examine the possible orientations more exactly. With the "partite" maximal degrees $\Delta_{1}(\mathcal{H}), \Delta_{2}(\mathcal{H}), \ldots, \Delta_{k}(\mathcal{H})$ from Lemma 3.3 we obtain, similarly as in Lemma 3.2:

Lemma 3.4. Let $\mathcal{H}=(V, \mathcal{E})$ be a $k$-partite $k$-uniform hypergraph with parts $V_{1}, V_{2}, \ldots, V_{k}$. For any $v \in V=V_{1} \uplus V_{2} \uplus \cdots \uplus V_{k}$, let $i(v)$ denote the index with $v \in V_{i(v)}$.

$$
\text { If } \frac{L_{1}}{\Delta_{1}(\mathcal{H})}+\frac{L_{2}}{\Delta_{2}(\mathcal{H})}+\cdots+\frac{L_{k}}{\Delta_{k}(\mathcal{H})}>1-\frac{1}{|\mathcal{E}|} \text {, for some nonnegative integers } L_{1}, L_{2}, \ldots, L_{k} \text {, }
$$
then there exists an orientation $\varphi: \mathcal{E} \longrightarrow V$ such that $d^{\varphi}(v) \leq L_{i(v)}$ for all $v \in V$.

Proof. The proof works exactly as that of Lemma 3.2. We just have to construct a graph $B^{L_{1}, \ldots, L_{k}}$ with $L_{i}$ copies of the vertices in $V_{i}(i=1, \ldots, k)$ instead of the graph $B^{m}$. Hall's theorem is applicable in the modified proof as each subset $E \subseteq \mathcal{E}$ of edges in $\mathcal{H}$ "meets" at least $|E| / \Delta_{i}(\mathcal{H})$ vertices in $V_{i}$, and this means that each subset $\bar{E} \subseteq \overline{\mathcal{E}}$ of new vertices has at least

$$
\frac{L_{1}|\bar{E}|}{\Delta_{1}(\mathcal{H})}+\frac{L_{2}|\bar{E}|}{\Delta_{2}(\mathcal{H})} \cdots+\frac{L_{k}|\bar{E}|}{\Delta_{k}(\mathcal{H})}>\left(1-\frac{1}{|\mathcal{E}|}\right)|\bar{E}| \geq|\bar{E}|-1
$$

neighbors in $B^{L_{1}, \ldots, L_{k}}$.

Now, it is easy to combine our results with Theorem 2.2. We obtain a series of upper bounds for the list chromatic number of $k$-partite $k$-uniform hypergraphs. The first one follows with the help of Lemma 3.2 and generalizes [AlTa, Theorem 3.4]: 
Theorem 3.5. $k$-partite $k$-uniform hypergraphs $\mathcal{H}$ are $r$-list colorable for

$$
r:=\lceil L(\mathcal{H})+1\rceil=\lfloor\check{L}(\mathcal{H})+2\rfloor .
$$

With Lemma 3.3 we obtain the following corollary to Theorem 3.5:

Corollary 3.6. $k$-partite $k$-uniform hypergraphs $\mathcal{H}=\left(V_{1} \uplus V_{2} \uplus \cdots \uplus V_{k}, \mathcal{E}\right)$ with "partite" maximal degrees $\Delta_{1}(\mathcal{H}), \Delta_{2}(\mathcal{H}), \ldots, \Delta_{k}(\mathcal{H})$ are $\left\lfloor\frac{1-1 /|\mathcal{E}|}{\sum_{i=1}^{k} 1 / \Delta_{i}(\mathcal{H})}+2\right\rfloor$-list colorable.

Proof. We combine the upper bound from Lemma 3.3 with

$$
\check{L}(\mathcal{H}) \leq\left(1-\frac{1}{\mathcal{E}(\mathcal{H}) \mid} L(\mathcal{H})\right.
$$

which follows from the fact that for partial hypergraph $H \leq \mathcal{H}$

$$
\frac{|\mathcal{E}(H)|-1}{|V(H)|}=\left(1-\frac{1}{|\mathcal{E}(H)|}\right) \frac{|\mathcal{E}(H)|}{|V(H)|} \leq\left(1-\frac{1}{|\mathcal{E}(\mathcal{H})|}\right) \frac{|\mathcal{E}(H)|}{|V(H)|} .
$$

If we apply this corollary to " $K_{2,3}$ minus one edge", as 2-partite 2-uniform hypergraph, it tells us that this graph is 2-list colorable. This would not follow from the the weaker upper bound $\left\lceil\frac{1}{\sum_{i=1}^{k} 1 / \Delta_{i}(\mathcal{H})}+1\right\rceil$ based on the expression $\lceil L(\mathcal{H})+1\rceil$ in Theorem 3.5 alone. The small improvement $\check{L}(\mathcal{H}) \leq\left(1-\frac{1}{|\mathcal{E}(\mathcal{H})|}\right) L(\mathcal{H})$ in the proof of the corollary makes a difference, even though $\lceil L(\mathcal{H})\rceil=\lfloor\check{L}(\mathcal{H})+1\rfloor$.

Now we apply Lemma 3.4, and obtain (again based on Theorem 2.2):

Theorem 3.7. Let $\mathcal{H}=(V, \mathcal{E})$ be a k-partite $k$-uniform hypergraph with parts $V_{1}, V_{2}, \ldots, V_{k}$. For any $v \in V=V_{1} \uplus V_{2} \uplus \cdots \uplus V_{k}$, let $i(v)$ denote the index with $v \in V_{i(v)}$.

$$
\text { If } \frac{L_{1}}{\Delta_{1}(\mathcal{H})}+\frac{L_{2}}{\Delta_{2}(\mathcal{H})}+\cdots+\frac{L_{k}}{\Delta_{k}(\mathcal{H})}>1-\frac{1}{|\mathcal{E}|} \text {, for some nonnegative integers } L_{1}, L_{2}, \ldots, L_{k} \text {, }
$$
then $\mathcal{H}$ is $\ell$-list colorable for $\ell:=\left(L_{i(v)}+1\right)_{v \in V}$.

If we apply this theorem to

$$
L_{1}=L_{2}=\ldots=L_{k}:=\left\lfloor\frac{1-1 /|\mathcal{E}|}{1 / \Delta_{1}(\mathcal{H})+\cdots+1 / \Delta_{k}(\mathcal{H})}+1\right\rfloor>\frac{1-1 /|\mathcal{E}|}{1 / \Delta_{1}(\mathcal{H})+\cdots+1 / \Delta_{k}(\mathcal{H})},
$$

it leads to the same upper bound as in Corollary 3.6.

\section{A Paintability Combinatorial Nullstellensatz}

We introduced paintability based on our game of Mr. Paint and Mrs. Correct already in [Scha2] for graphs. It is obvious how to generalize this game to hypergraphs, but it can even be generalized to polynomials $0 \neq P \in \mathcal{R}\left[X_{V}\right]:=\mathcal{R}\left[X_{v} \mid v \in V\right]$ in finitely many variables over integral domains $R$. The variables $X_{v}$ play the role of the vertices, 
and the initial stacks $S_{v}$ of $\ell_{v}-1$ erasers are assigned to them. The idea is that Mr. Paint substitutes in the $i^{\text {th }}$ round a new symbolic variable $T_{i}$ for some of the variables

$S_{v}$

$T_{i}$ $X_{v}$, instead of coloring some vertices $v$ with the $i^{\text {th }}$ color. Mrs. Correct has then to use up some of the erasers in order to keep the polynomial different from zero, by partially undoing the substitution. We say the polynomial is $\ell$-paintable $\left(\ell=\left(\ell_{v}\right)_{v \in V}\right)$ if she always can achieve this, i.e., no matter how Mr. Paint plays, she can use the erasers in such a way that after finitely many rounds all $X_{v}$ are replaced without making the polynomial zero. To make this more precise we will need the following definitions:

Definition 4.1 (Cut Off Operator). Assume $U \subseteq V, P \in \mathcal{R}\left[X_{V}\right]$, and let $T \notin \mathcal{R}\left[X_{V}\right]$ be a symbolic variable. We write

$$
P \backslash U=P \backslash_{T} U:=\left.P\right|_{\substack{x_{v}=T \\ v \in U}} \in \mathcal{R}^{\prime}\left[X_{V \backslash U}\right]:=(\mathcal{R}[T])\left[X_{V \backslash U}\right]
$$

for the polynomial over $\mathcal{R}^{\prime}:=\mathcal{R}[T]$ that we obtain from $P$ by substituting the "color" $T$ for all variables $X_{v}$ with $v \in U$. Which symbolic variable $T$ we choose does not play a role, but it has to be new, chosen outside the current polynomial ring. For example,

$$
\left(P \backslash U_{1}\right) \backslash U_{2} \text { has to be read as }\left(P \backslash_{T_{1}} U_{1}\right) \backslash_{T_{2}} U_{2}
$$

with $T_{1} \notin \mathcal{R}\left[X_{V}\right]$ and $T_{2} \notin \mathcal{R}^{\prime}\left[X_{V \backslash U_{1}}\right]:=\left(\mathcal{R}\left[T_{1}\right]\right)\left[X_{V \backslash U_{1}}\right]$.

Definition 4.2 (Mounted Polynomial). A mounted polynomial $P^{\ell}$ is a pair $(P, \ell)$ of a polynomial $P \in \mathcal{R}\left[X_{V}\right]$ and a tuple $\ell \in \mathbb{Z}^{V}$. Usually $\ell \geq 1$, and we suggest to imagine a stack $S_{v}$ of $\ell_{v}-1 \geq 0$ "erasers" at each index $v \in V$. We treat $P^{\ell}$ as any usual polynomial; just, when we change the polynomial, we adapt the stacks of erasers in the natural way. For example, if $U \subseteq V$, then

$$
P^{\ell} \backslash U:=(P \backslash U)^{\left.\ell\right|_{V \backslash U}}
$$

We also introduce a new operator $\downarrow$ (down) which acts only on the stacks of erasers.

Definition 4.3 (Down Operator). For arbitrary sets $U$, we set

$$
P^{\ell} \downarrow U:=P^{\ell-1_{(U \cap V)}}, \quad \text { with } 1_{(U \cap V)}(v):= \begin{cases}1 & \text { if } v \in U \cap V, \\ 0 & \text { if } v \in V \backslash U,\end{cases}
$$

and abbreviate

$$
P^{\ell}\left\lfloor U_{1} \backslash U_{2}:=\left(P^{\ell}\left\lfloor U_{1}\right) \backslash U_{2}\right.\right.
$$

Now, paintability can be defined recursively as follows:

Definition 4.4 (Paintability). Let $\ell \in \mathbb{Z}^{V}$ and $P \in \mathcal{R}\left[X_{V}\right] . P$ is said to be $\ell$-paintable if the mounted polynomial $P^{\ell}$ is paintable in the following recursively defined sense:

(i) If $V=\varnothing$ then $P^{\ell}$ is paintable if and only if $P \neq 0$ (where $\ell$ is the empty tuple). 
(ii) If $V \neq \varnothing$ then $P^{\ell}$ is paintable if $\ell \geq 1$ and if each nonempty subset $V_{P} \subseteq V$ of indices contains a good subset $V_{C} \subseteq V_{P}$, i.e., a subset $V_{C} \subseteq V_{P}$ such that $P^{\ell}\left\lfloor V_{P} \backslash V_{C}\right.$ is paintable.

(Mr. Paint "paints" all variables $X_{v}$ with indices $v$ in $V_{P}$, so that one eraser from each stack $S_{v}$ with $v \in V_{P} \backslash V_{C}$ has to be used up by Mrs. Correct, in order to undo the suggested coloring (substitution) of the corresponding variables $X_{v}$.)

It is not hard to see that this generalizes paintability of hypergraphs $\mathcal{H}$. A partial coloring of a hypergraph $\mathcal{H}$ with symbolic variables is correct if and only if the corresponding partial substitution in the matrix polynomial $P_{A}$ does not annihilate $P_{A}$ (where $A$ is a zero row-sum incidence matrix of $\mathcal{H}$, and $P_{A}$ is defined in Equation (3)). Hence,

$$
\mathcal{H} \text { is } \ell \text {-paintable } \Longleftrightarrow P_{A} \text { is } \ell \text {-paintable } .
$$

That paintability generalizes list colorability was already described in the introduction. However, it can also be understood out of the more game-theoretic definitions in this section. Imagine that, during the game, Mr. Paint writes down the "colors" he suggests for the variable $X_{v}$ in a list $L_{v}$. Then, at the end of the game, the list $L_{v}$ has at most $\ell_{v}$ entries, since $\ell_{v}-1$ is the maximal number of rejections at $X_{v}$ (there are $\ell_{v}-1$ erasers at $X_{v}$ ), and $X_{v}$ is just "colored" with the last one in it. Hence, paintability may be seen as a dynamic version of list colorability, where the lists $L_{v}$ of symbolic variables are not completely fixed before the coloration process starts. If lists $L_{v}$ are fixed at the beginning and $\left|L_{v}\right| \geq \ell_{v}$, for all $v \in V$, then

$$
P \text { is } \ell \text {-paintable } \Longrightarrow P(x) \neq 0 \text { for an } x \in \prod_{v} L_{v} \text {. }
$$

The graph-theoretic examples [Scha2, Example 1.5] and [Zhu, Section 4] show that the converse is wrong. Therefore, if we only study lists $L_{v} \subseteq\left\{T_{1}, T_{2}, \ldots\right\}$ of symbolic variables, the following theorem is stronger than the Combinatorial Nullstellensatz 1.1, and can be used in place of it in the proof of Theorem 2.1. It does not contain degree restrictions either (because of Implication (35)).

Writing $P_{\delta}$ for the coefficient of $X^{\delta}:=\prod_{v \in V} X_{v}^{\delta_{v}}$ in $P \in \mathcal{R}\left[X_{V}\right]$, we provide:

Theorem 4.5. Let $P=\sum_{\delta \in \mathbb{N}^{V}} P_{\delta} X^{\delta} \in \mathcal{R}\left[X_{V}\right]$ and $\alpha \in \mathbb{N}^{V}$, then

$$
P_{\alpha} \neq 0 \Longrightarrow P \text { is }(\alpha+1) \text {-paintable. }
$$

In order to prove this, we will need the following generalization of [Scha3, Lemma 2.2] . With $\alpha+\mathbb{N}^{U}:=\left\{\alpha^{\prime} \geq \alpha \mid \alpha_{v}^{\prime}=\alpha_{v}\right.$ for all $\left.v \notin U\right\}$ and $1_{u}:=1_{\{u\}}=\left(\delta_{u, v}\right)_{v \in V}$ it holds:

Lemma 4.6. Let $P=\sum_{\delta \in \mathbb{N}^{V}} P_{\delta} X^{\delta} \in \mathcal{R}\left[X_{V}\right]$ be a polynomial and $\alpha \in \mathbb{N}^{V}$. Let $V_{P} \subseteq V$ be nonempty and $u \in V_{P}$. Then:

(i) $\left(\alpha-1_{u}\right)+\mathbb{N}^{V_{P}}=\alpha+\mathbb{N}^{V_{P}} \uplus\left(\alpha-1_{u}\right)+\mathbb{N}^{V_{P} \backslash u}$. 


$$
\begin{aligned}
& \text { (ii) } \sum_{\delta \in\left(\alpha-1_{u}\right)+\mathbb{N}^{V_{P}}} P_{\delta}=\sum_{\delta \in \alpha+\mathbb{N}^{V_{P}}} P_{\delta}+\sum_{\delta \in\left(\alpha-1_{u}\right)+\mathbb{N}^{V_{P} \backslash u}} P_{\delta} \text {. } \\
& \text { (iii) } \sum_{\delta \in \alpha+\mathbb{N}^{V_{P}}} P_{\delta} \neq 0 \Longrightarrow \sum_{\delta \in\left(\alpha-1_{u}\right)+\mathbb{N}^{V_{P}}} P_{\delta} \neq 0 \quad \vee \sum_{\delta \in\left(\alpha-1_{u}\right)+\mathbb{N}^{V_{P} \backslash u}} P_{\delta} \neq 0 \text {. } \\
& \text { (iv) } \sum_{\delta \in \alpha+\mathbb{N}^{V_{P}}} P_{\delta} \neq 0 \Longrightarrow\left\{\begin{array}{l}
\text { There is an } \alpha^{\prime} \leq \alpha \text { and } a V_{C} \subseteq V_{P} \text { such that: }\left.\alpha^{\prime}\right|_{V_{C}} \equiv 0, \\
\alpha_{v}^{\prime}<\alpha_{v} \text { for all } v \in V_{P} \backslash V_{C} \text {, and } \sum_{\delta \in \alpha^{\prime}+\mathbb{N}^{V_{C}}} P_{\delta} \neq 0
\end{array}\right.
\end{aligned}
$$

Proof. The elements $\sigma$ of the set $\left(\alpha-1_{u}\right)+\mathbb{N}^{V_{P}}$ on the left side of Equation $(i)$ fulfill $\sigma_{u} \geq \alpha_{u}-1$. On the right side, we simply distinguish between elements with $\sigma_{u}>\alpha_{u}-1$ and elements with $\sigma_{u}=\alpha_{u}-1$.

Furthermore, Equation $(i)$ implies Equation (ii), which entails Implication (iii).

In order to prove Implication $(i v)$, we may iteratively use Implication (iii) to produce sequences

$$
\alpha=: \alpha^{0} \geqslant \alpha^{1} \ngtr \cdots \geqslant \alpha^{t} \geq 0 \quad \text { and } \quad V_{P}=: V_{C}^{0} \supseteq V_{C}^{1} \supseteq \cdots \supseteq V_{C}^{t}
$$

with the property

$$
\sum_{\delta \in \alpha^{i}+\mathbb{N}^{v} V_{\delta}^{i}} P_{\delta} \neq 0 \quad \text { for } i=0,1, \ldots, t
$$

Note that

$$
\left.\alpha^{t}\right|_{V_{C}^{t}} \equiv 0
$$

if and only if the sequence of componentwise nonnegative $\alpha^{i}$ in (31) can no longer be extended through application of Implication (iii); hence, in this case Implication (iv) holds, if we set

$$
\alpha^{\prime}:=\alpha^{t} \quad \text { and } \quad V_{C}:=V_{C}^{t} .
$$

With this, the proof of Theorem 4.5 is based on the same idea as our purely combinatorial proof of Alon and Tarsi's Theorem [Scha3, Theorem 2.1]. However, we also need that we may focus on one homogeneous component $H$ of $P \in \mathcal{R}\left[X_{V}\right]$ when we substitute symbolic variables $T_{1}, T_{2}, T_{3}, \ldots$ If $x=\left(x_{v}\right)_{v \in V} \in\left\{T_{j}, X_{v} \mid j \in \mathbb{N}, v \in V\right\}^{V}$, then

$$
H(x) \neq 0 \quad \Longrightarrow \quad P(x) \neq 0
$$

since $H(x)$ is still a homogenous component of $P(x)$, if we view $H(x)$ and $P(x)$ as polynomials in the variables $T_{j}$ and $X_{v}(j \in \mathbb{N}, v \in V)$.

Proof of Theorem 4.5. Let a nonempty subset $V_{P} \subseteq V$ be given. In view of Implication (35), we may assume that $P$ is homogeneous of degree

$$
\operatorname{deg}(P)=\operatorname{deg}\left(X^{\alpha}\right),
$$


so that

$$
\sum_{\delta \in \alpha+\mathbb{N} V_{P}} P_{\delta}=P_{\alpha} \neq 0
$$

and we can apply Lemma $4.6(i v)$. This yields a potentially good subset $V_{C} \subseteq V_{P}$ and a tuple $\alpha^{\prime} \leq \alpha$. We substitute $T$ for all variables $X_{v}$ with $v \in V_{C}$ in $P$, and obtain the polynomial

$$
P \backslash V_{C} \in \mathcal{R}^{\prime}\left[X_{V \backslash V_{C}}\right] \quad \text { with } \quad \mathcal{R}^{\prime}:=\mathcal{R}[T]
$$

We know that

$$
\left(P \backslash V_{C}\right)_{\alpha^{\prime \prime}} \neq 0 \quad \text { for } \quad \alpha^{\prime \prime}:=\left.\alpha^{\prime}\right|_{V \backslash V_{C}},
$$

since even

$$
\left.\left(P \backslash_{T} V_{C}\right)_{\alpha^{\prime \prime}}\right|_{T=1} \stackrel{4.2}{=}\left(\left.P\right|_{\substack{x_{v}=1 \\ v \in V_{C}}}\right)_{\alpha^{\prime \prime}}=\sum_{\delta \in \alpha^{\prime}+\mathbb{N}^{V_{C}}} P_{\delta} \stackrel{4.6}{\neq} 0,
$$

as $\left.\alpha^{\prime}\right|_{V_{C}} \stackrel{4.6}{\equiv} 0$. Using an induction argument, it follows that $P \backslash V_{C}$ is $\left(\alpha^{\prime \prime}+1\right)$-paintable. Hence,

$$
P^{\left(\alpha^{\prime}+1\right)} \backslash V_{C}=\left(P \backslash V_{C}\right)^{\left(\alpha^{\prime \prime}+1\right)}
$$

is paintable, and so is $P^{(\alpha+1)} \downarrow V_{P} \backslash V_{C}$, as $\alpha_{v}^{\prime}<\alpha_{v}$ for all $v \in V_{P} \backslash V_{C}$. This means, in view of Definition 4.4 , that $P$ is $(\alpha+1)$-paintable.

Note that it was necessary to use symbolic variables in Theorem 4.5, a similar version where we allow Mr. Paint to use elements of the ground $\operatorname{ring} \mathcal{R}$ does not hold. The polynomial $P:=X_{1}+X_{2}-2 \in \mathbb{Z}\left[X_{1}, X_{2}\right]$ with one eraser at $X_{1}(\alpha:=(1,0)$ in Theorem 4.5$)$ is a counterexample. Mr. Paint may play as follows:

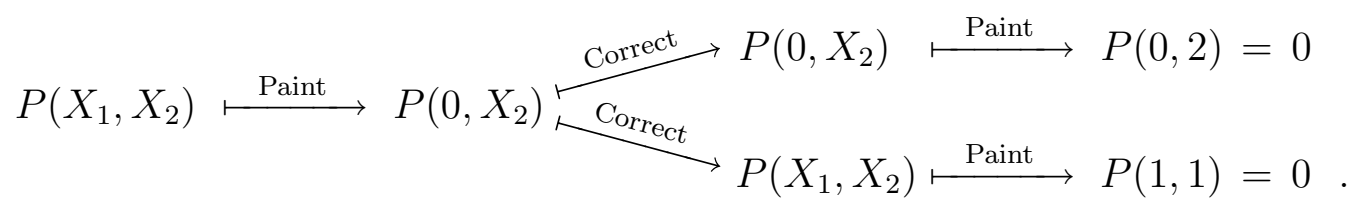

\section{Acknowledgement:}

We are grateful to Ayub Khan for his help. Furthermore, the author gratefully acknowledges the support provided by the King Fahd University of Petroleum and Minerals.

\section{References}

[Al] N. Alon: Restricted Colorings of Graphs.

In "Surveys in combinatorics, 1993", London Math. Soc. Lecture Notes Ser. 187, Cambridge Univ. Press, Cambridge 1993, 1-33.

[Al2] N. Alon: Combinatorial Nullstellensatz.

Combin. Probab. Comput. 8, No. 1-2 (1999), 7-29. 
[AlTa] N. Alon, M. Tarsi: Colorings and Orientations of Graphs. Combinatorica 12 (1992), 125-134.

[AlTa2] N. Alon, M. Tarsi: A Nowhere-Zero Point in Linear Mappings. Combinatorica 9 (1989), 393-395.

[ERT] P. Erdős, A. L. Rubin, H. Taylor: Choosability in Graphs.

Proc. West Coast Conf. on Combinatorics, Graph Theory and Computing, Congressus Numerantium XXVI (1979), 125-15\%.

[HKS] J.Hladký, D. Král', U. Schauz: Brooks' Theorem via the Alon-Tarsi Theorem. Discrete Mathematics 310 (2010), 3426-3428.

[KTV] J. Kratochvl, Zs. Tuza, M. Voigt:

New Trends in the Theory of Graph Colorings: Choosability and List Coloring. In: R.L. Graham et al., Editors, Contemporary Trends in Discrete Mathematics. DIMACS Ser. Discr. Math. Theo. Comp. Sci. 49, Amer. Math. Soc. (1999), 183197.

[RaWe] R. Ramamurthi, D. B. West:

Hypergraph Extension of the Alon-Tarsi List Coloring Theorem. Combinatorica 25, No. 3, (2005), 355-366.

[Scha1] U. Schauz: Algebraically Solvable Problems: Describing Polynomials as Equivalent to Explicit Solutions The Electronic Journal of Combinatorics 15 (2008), \#R10.

[Scha2] U.Schauz: Mr. Paint and Mrs. Correct. The Electronic Journal of Combinatorics 16/1 (2009), \#R77.

[Scha3] U.Schauz: Flexible Color Lists Alon and Tarsi's Theorem, and Time Scheduling with Unreliable Participants. The Electronic Journal of Combinatorics 17/1 (2010), \#R13.

[Tu] Zs. Tuza: Graph Colorings With Local Constraints - A Survey. Discuss. Math. Graph Theory, 17, (1997), 161228.

[Viz] V. G. Vizing: Coloring the vertices of a graph in prescribed colors (in Russian). Diskret. Analiz. No. 29, Metody Diskret. Anal. v. Teorii Kodov i Shem 101 (1976), 3-10.

[Zhu] Xuding Zhu: On-Line List Colouring of Graphs. The Electronic Journal of Combinatorics 16/1 (2009), \#R12\%. 